\title{
CRITICAL EXPONENTS OF INVARIANT RANDOM SUBGROUPS IN NEGATIVE CURVATURE
}

\author{
ILYA GEKHTMAN AND ARIE LEVIT
}

\begin{abstract}
Let $X$ be a proper geodesic Gromov hyperbolic metric space and let $G$ be a cocompact group of isometries of $X$ admitting a uniform lattice. Let $d$ be the Hausdorff dimension of the Gromov boundary $\partial X$. We define the critical exponent $\delta(\mu)$ of any discrete invariant random subgroup $\mu$ of the locally compact group $G$ and show that $\delta(\mu)>\frac{d}{2}$ in general and that $\delta(\mu)=d$ if $\mu$ is of divergence type. Whenever $G$ is a rank-one simple Lie group with Kazhdan's property $(T)$ it follows that an ergodic invariant random subgroup of divergence type is a lattice. One of our main tools is a maximal ergodic theorem for actions of hyperbolic groups due to Bowen and Nevo.
\end{abstract}

\section{INTRODUCTION}

Let $X$ be a proper geodesic Gromov hyperbolic metric space and let $\operatorname{Isom}(X)$ denote its group of isometries. Fix a basepoint $o \in X$. The critical exponent $\delta(\Gamma)$ of a discrete subgroup $\Gamma$ of $\operatorname{Isom}(X)$ is given by

$$
\delta(\Gamma)=\inf \left\{s: \sum_{\gamma \in \Gamma} e^{-s d_{X}(o, \gamma o)}<\infty\right\} .
$$

The subgroup $\Gamma$ is of divergence type if the above series diverges at the critical exponent. A discrete subgroup of $\operatorname{Isom}(X)$ is a uniform lattice if it acts cocompactly on $X$. A uniform lattice is always of divergence type and its critical exponent is equal to the Hausdorff dimension $\operatorname{dim}_{\mathrm{H}}(\partial X)$ of the Gromov boundary of $X$.

For example, if $X$ is the $n$-dimensional hyperbolic space $\mathbb{H}^{n}$ and $\Gamma$ is a uniform lattice in $\operatorname{Isom}\left(\mathbb{H}^{n}\right)$ then $\delta(\Gamma)=\operatorname{dim}_{H}\left(\partial \mathbb{H}^{n}\right)=n-1$.

An invariant random subgroup of a locally compact group $G$ is a conjugation invariant Borel probability measure on the Chabauty space of its closed subgroups. Lattices as well as normal subgroups of lattices provide examples of invariant random subgroups.

Theorem 1.1. Let $G$ be a closed non-elementary subgroup of $\operatorname{Isom}(X)$ acting cocompactly on $X$ and admitting a uniform lattice. Let $\mu$ be an invariant random subgroup of $G$ such that $\mu$-almost every closed subgroup of $G$ is discrete and infinite. Then

(1) $\delta(H)>\frac{1}{2} \operatorname{dim}_{H}(\partial X)$, and

(2) if $H$ has divergence type then $\delta(H)=\operatorname{dim}_{H}(\partial X)$

for $\mu$-almost every closed subgroup $H$.

Since $\delta(\Gamma)=\liminf _{R \rightarrow \infty} \frac{1}{R} \ln \left|\Gamma o \cap B_{X}(o, R)\right|$, the conclusion of part (1) of Theorem 1.1 says that the orbits of discrete invariant random subgroups of $G$ are in a certain sense "large". 
Patterson constructed discrete subgroups of Isom $\left(\mathbb{H}^{n}\right)$ having full limit sets, arbitrary small positive critical exponents and being of convergence as well as of divergence type Pat83. By our main result such discrete subgroups cannot be assigned positive measure by any invariant random subgroup of $\operatorname{Isom}\left(\mathbb{H}^{n}\right)$.

In particular Theorem 1.1 applies to the action of a word hyperbolic group $G$ on its Cayley graph. This viewpoint is useful and gives a sharper result even if $G$ happens to be a uniform lattice in a rank one simple linear group, since in general the exact value of the critical exponent changes under a quasi-isometry.

If $G$ is a rank one simple linear group over a local field then it admits a uniform lattice and any non-atomic invariant random subgroup of $G$ is discrete and infinite $\mathrm{ABB}^{+} 17$, GL17, so that these assumptions of Theorem 1.1 are satisfied automatically. We obtain the following characterization of invariant random subgroups of divergence type.

Corollary 1.2. Let $G$ be a rank-one simple Lie group and $\mu$ be a non-atomic invariant random subgroup of $G$. Then $\mu$-almost every discrete subgroup $\Gamma$ of $G$ has divergence type if and only if the geodesic flow on $\Gamma \backslash G$ is ergodic with respect to the Haar measure class.

Kesten's theorem for invariant random subgroups of rank-one simple Lie groups. Consider the case where $G$ is a rank-one simple Lie group and $X$ is the associated symmetric space. Let $\Gamma$ be a discrete torsion-free subgroup of $G$ so that the quotient $\Gamma \backslash X$ is a locally symmetric Riemannian manifold.

Let $\lambda_{0}(\Gamma \backslash X)$ denote the bottom of the spectrum of the Laplace-Beltrami operator on $\Gamma \backslash X$. The two quantities $\lambda_{0}(\Gamma \backslash X)$ and $\delta(\Gamma)$ stand in the following quadratic relation Els73a, Els73b, Els74, Pat76, Sul87, Leu04]

$\lambda_{0}(\Gamma \backslash X)=\left\{\begin{array}{ll}\frac{1}{4} d(X)^{2} & \text { if } 0 \leq \delta(\Gamma) \leq \frac{1}{2} d(X) \\ \delta(\Gamma)(d(X)-\delta(\Gamma)) & \text { if } \frac{1}{2} d(X) \leq \delta(\Gamma) \leq d(X)\end{array}\right.$ where $d(X)=\operatorname{dim}_{\mathrm{H}}(\partial X)$.

For instance $\lambda_{0}(X)=\frac{1}{4} \operatorname{dim}_{\mathrm{H}}(\partial X)^{2}$. Moreover $\Gamma$ has critical exponent equal to $\operatorname{dim}_{\mathrm{H}}(\partial X)$ if and only if $\Gamma$ is co-amenable.

Corollary 1.3. Let $G$ be a rank-one simple Lie group and $\mu$ be a non-atomic invariant random subgroup of $G$ so that $\mu$-almost every subgroup is torsion-free. Then $\lambda_{0}(\Gamma \backslash X)<\lambda_{0}(X)$ holds $\mu$-almost surely.

We know from Theorem 1.1 that $\mu$-almost every subgroup is non-elementary and is in particular non-amenable. Therefore our Corollary 1.3 is a natural generalization of the main result of Abért, Glasner and Virág AGV14 to invariant random subgroups of rank one simple Lie groups with respect to the geometric Laplacian.

It is known that higher-rank symmetric spaces satisfy Kesten's theorem for irreducible invariant random subgroups in a much stronger sense. Indeed, every non-atomic irreducible invariant random subgroup $\mu$ in a higher-rank Lie group is co-amenable [SZ94, $\mathrm{ABB}^{+} 17$, HT16] and so $\lambda_{0}(\Gamma \backslash X)=0$ holds $\mu$-almost surely. Therefore our Corollary 1.3 completes the picture for all semisimple Lie groups.

Hyperbolic spaces with Kazhdan isometry groups. Whenever $\operatorname{Isom}(X)$ has Kazhdan's property $(T)$ our main result admits an interesting application, which can be regarded as a certain conditional rank one analogue of the celebrated theorem of Stuck and Zimmer [SZ94]. 
Corollary 1.4. If $G$ is a rank-one simple Lie group with Kazhdan's property (T) then any non-atomic ergodic invariant random subgroup of $G$ of divergence type is essentially a lattice.

Similarly, if $G$ has Kazhdan's property $(T)$ and is either a hyperbolic group acting on its Cayley graph or a uniform lattice in $\operatorname{Isom}(X)$ for some $\mathrm{CAT}(-1)$-space $X$ then any invariant random subgroup of $G$ is supported on finite index subgroups.

Corollary 1.4 follows directly from part (2) of Theorem 1.1 together with Corlette's work Cor90 on discrete subgroups of rank one Lie groups with property $(T)$ and its generalization by Coulon-Dal'bo-Sambusetti CDS17. Indeed, if $G$ has property $(T)$ then there exists a certain threshold value $0<\delta_{c}<\operatorname{dim}_{H}(\partial X)$ such that every discrete subgroup $\Gamma$ with $\delta(\Gamma)>\delta_{c}$ must be a lattice.

Stabilizers of probability measure preserving actions. Invariant random subgroups can be used towards the study of probability measure preserving actions by considering stabilizers of random points.

Corollary 1.5. Let $G$ be a closed non-elementary subgroup of $\operatorname{Isom}(X)$ acting cocompactly on $X$ and admitting a uniform lattice. Let $(Z, \mu)$ be a Borel space with a probability measure preserving action of $G$. If the stabilizer $G_{z}$ of $\mu$-almost every point $z \in Z$ is discrete and infinite then $\mu$-almost surely $\delta\left(G_{z}\right)>\frac{\operatorname{dim}_{\mathrm{H}}(\partial X)}{2}$.

By inducing invariant random subgroups from a lattice we obtain the following.

Corollary 1.6. Assume that $\operatorname{Isom}(X)$ is non-elementary and admits a uniform lattice. Let $\Gamma$ be any lattice in $\operatorname{Isom}(X)$ and $(Z, \mu)$ be any Borel space with a probability measure preserving action of $\Gamma$. Then the stabilizer $\Gamma_{z}$ of $\mu$-almost every point $z \in Z$ is either finite or satisfies $\delta\left(\Gamma_{z}\right)>\frac{\operatorname{dim}_{H}(\partial X)}{2}$.

We emphasize that the lattice $\Gamma$ considered in Corollary 1.6 need not be uniform. For example, as an abstract group $\Gamma$ can be a finitely generated free group or the fundamental group of any finite volume hyperbolic manifold.

A few words about our proofs. Let $\Gamma$ be a uniform lattice in $\operatorname{Isom}(X)$ with critical exponent $\delta(\Gamma)$. Consider some fixed hyperbolic element $h \in \operatorname{Isom}(X)$. Our proof relies on the essentially elementary observation that a partial Poincare series evaluated over the orbit of $h$ under conjugation by elements of the lattice $\Gamma$ behaves like a Poincare series of exponent $2 \delta$. This strategy relies on hyperbolicity. Of course, unless $h$ belongs to a normal subgroup, there is no reason for these conjugates to be contained in any particular discrete subgroup. This complicates our proofs considerably. To overcome this problem we use a maximal ergodic theorem for hyperbolic groups, due to Bowen-Nevo [BN13, to obtain a quantitative estimate for the recurrence of the invariant random subgroup to a neighborhood of the orbit in question. Finally we use quasiconformal densities and Patterson-Sullivan theory to deduce sharper results for divergence type groups. The paper includes an appendix on measurability issues of the above notions in the Chabauty topology.

Previous work. A normal subgroup of a lattice is a very special kind of an invariant random subgroup. Our work extends results known in that setting. Theorem 1.1 is originally due to Jaerisch [Jae15] in the case of normal subgroups of Kleinian groups. This was later generalized by Matsuzaki-Yabuki-Jaerisch MYJ15 to deal with normal subgroups of discrete isometry groups of proper Gromov hyperbolic 
spaces. Arzhantseva and Cashen [AC18 have recently generalized MYJ15] to normal subgroups of finitely generated groups acting on proper geodesic spaces with a strongly contracting element. This class includes rank-one actions on CAT(0) spaces as well as the mapping class group action on the Teichmüller space equipped with the Teichmüller metric.

We recall Kesten's theorem Kes59 and its recent generalization by Abért, Glasner and Virág AGV14 to invariant random subgroups. Let $\Gamma$ be any finitely generated group and $S \subset \Gamma$ be some symmetric finite generating set. Let $\lambda_{0}(\Gamma)$ denote the bottom of the spectrum of the combinatorial Laplacian on the Cayley graph of $\Gamma$ with respect to $S$. Similarly $\lambda_{0}(H \backslash \Gamma)$ is defined for any quotient of the Cayley graph by a subgroup $H \leq \Gamma$. Given an invariant random subgroup $\mu$ of $\Gamma$ it is shown in AGV14 that $\lambda_{0}(H \backslash \Gamma)<\lambda_{0}(\Gamma)$ if and only if the subgroup $H$ is non-amenable $\mu$-almost surely. Kesten's classical theorem is the special case where $\mu$ is supported on some normal subgroup $N$ of $\Gamma$. Our Corollary 1.3 can be seen as a generalization of this, and is based on completely different methods.

Cannizzo showed that the action of an invariant random subgroup of a countable group on the Poisson boundary of its enveloping group is almost surely conservative Can14, Theorem 1.2.2]. Grigorchuk, Kaimanovich and Nagnibeda showed that if $\Gamma$ is a free group and $H$ is a subgroup so that $\delta(H)<\frac{\delta(\Gamma)}{2}$ with respect to a free generating set, then the action of $H$ on the boundary of $\Gamma$ is dissipative GKN12, Theorem 4.2]. The analogous result for discrete Fuchsian groups where the critical exponent taken with respect to the hyperbolic plane metric is due to Patterson Pat77] and Matsuzaki Mat05. Combining these results provides a different proof of a non-strict variant of part (1) of our Theorem 1.1 for these particular actions of free groups and surface groups. We remark that Pat77. uses spectral methods while the approach of GKN12 is combinatorial, so that these proofs appear to be difficult to generalize to the general Gromov hyperbolic setting.

Acknowledgements. We would like to thank Mikolaj Fraczyk for pointing out the analogy with Kesten's theorem and leading us to include Corollary 1.3. We would like to thank Amos Nevo for useful remarks and suggestions concerning the ergodic theorem for hyperbolic groups. We would like to thank Tushar Das and David Simmons for illuminating remarks about critical exponents, especially alerting us to Patterson's construction of Kleinian groups with full limit set and arbitrarily small critical exponent. The first author is partially supported by NSF grant DMS-1401875.

\section{The CRItical exponent in Gromov hyperbolic SPACES}

Let $X$ be a proper geodesic Gromov hyperbolic metric space. We recall some basic notions regarding the Gromov boundary, the Poincare series and the critical exponent.

The Gromov boundary. A geodesic metric space $X$ is Gromov hyperbolic if all geodesic triangles with endpoints in $X$ are $\delta$-thin for some constant $\delta>0$. The Gromov boundary $\partial X$ of a proper geodesic Gromov hyperbolic space $X$ is the set of all geodesic rays, with two rays of finite Hausdorff distance being identified.

Ideal geodesic triangles with endpoints in $\partial X$ are also $\delta^{\prime}$-thin for some constant $\delta^{\prime}>0$ depending on $\delta$. In particular any two geodesic lines having the same 
endpoints either in $X$ or in $\partial X$ are within Hausdorff distance $\delta^{\prime}$ of each other [̄sh02. Denote

$$
\partial^{2} X=(\partial X \times \partial X) \backslash \text { Diag }
$$

so that $\partial^{2} X$ consists of all ordered pairs of distinct points on the boundary of $X$. For every $\left(x^{-}, x^{+}\right) \in \partial^{2} X$ there is a bi-infinite geodesic $l$ in $X$ with $l(-\infty)=x^{-}$ and $l(\infty)=x^{+}$.

A visual metric $\rho$ on the Gromov boundary $\partial X$ with parameter $a>0$ and basepoint $o \in X$ is any metric satisfying

$$
k_{1} a^{-d_{X}\left(o, l_{\xi_{1}, \xi_{2}}(\mathbb{R})\right)} \leq \rho\left(\xi_{1}, \xi_{2}\right) \leq k_{2} a^{-d_{X}\left(o, l_{\xi_{1}, \xi_{2}}(\mathbb{R})\right)}
$$

for some constants $k_{1}, k_{2}>0$ and every pair of points $\xi_{1}, \xi_{2} \in \partial X$, where $l_{\xi_{1}, \xi_{2}}$ : $\mathbb{R} \rightarrow X$ is any geodesic line with end points $l_{\xi_{1}, \xi_{2}}(-\infty)=\xi_{1}$ and $l_{\xi_{1}, \xi_{2}}(\infty)=\xi_{2}$. A visual metric $\rho_{a}$ on $\partial X$ exists for any value of the parameter $a$ within the range $a^{\prime}>a>1$, where $a^{\prime}>1$ is a constant depending only on $\delta$.

The Hausdorff dimension of the metric $\rho_{a}$ is equal to $\ln (a) d(X)$ for some constant $d(X)$ independent of the parameter $a$ and the basepoint $o \in X$. By a slight abuse of notation, the Hausdorff dimension of the Gromov boundary $\partial X$ is defined as $\operatorname{dim}_{H}(\partial X)=d(X)$. It is known that $\operatorname{dim}_{H}(\partial X)>0$ whenever $|\partial X|>2$ and that $\operatorname{dim}_{H}(\partial X)<\infty$ whenever Isom $(X)$ admits a uniform lattice.

The Hausdorff dimension of the Gromov boundary of the $n$-dimensional hyperbolic space is equal to its topological dimension $\operatorname{dim}_{\text {top }}\left(\partial \mathbb{H}^{n}\right)=n-1$. We point out that in general the Hausdorff and the topological dimensions of the Gromov boundary may be different. In fact Bonk and Kleiner BK02 proved that if $X$ is a $\mathrm{CAT}(-1)$-space not isometric to the $n$-dimensional hyperbolic space $\mathbb{H}^{n}$ for some $n$ then the strict inequality $d(X)>\operatorname{dim}_{\text {top }}(\partial X)$ holds.

The Gromov compactification of the metric space $X$ is denoted $\bar{X}=X \cup \partial X$. The group of isometries $\operatorname{Isom}(X)$ is locally compact and acts continuously on $\bar{X}$.

The Poincare series and the critical exponent. Let $\Gamma$ be any discrete subgroup of Isom $(X)$. The Poincare series of $\Gamma$ at the exponent $s \geq 0$ and with respect to the basepoint $o \in X$ is given by

$$
\mathcal{P}_{\Gamma}(s)=\sum_{\gamma \in \Gamma} e^{-s d_{X}(o, \gamma o)} .
$$

The critical exponent of $\Gamma$ is the infimum over all exponents $s$ such that $\mathcal{P}_{\Gamma}(s)$ converges. It is independent of the choice of basepoint. It is easy to see that

$$
\delta(\Gamma)=\liminf _{R \rightarrow \infty} \frac{1}{R} \ln \left|\Gamma o \cap B_{X}(o, R)\right| .
$$

The discrete group $\Gamma$ is said to be of divergence type if $\mathcal{P}_{\Gamma}(\delta(\Gamma))$ diverges, and of convergence type otherwise. If $\Gamma$ is a uniform lattice then it is necessarily of divergence type [Coo93, Corollary 7.3]. A discrete group of divergence type is clearly infinite.

The limit set $\Lambda(\Gamma)$ is the set of all accumulation points in the Gromov boundary $\partial X$ of some orbit of $\Gamma$ in $X$. The subgroup $\Gamma$ is non-elementary if $\Lambda(\Gamma)$ has more than two points. If $\Gamma$ is non-elementary then $\delta(\Gamma)>0$.

The radial limit set $\Lambda_{r}(\Gamma) \subset \Lambda(\Gamma)$ consists of the ideal points $\alpha \in \partial X$ such that there is a geodesic ray converging to $\alpha$ and intersecting non-trivially infinitely many $\Gamma$-translates of the ball $B_{X}(o, R)$ for some $R>0$. It is known by the work of 
Bishop-Jones [BJ97, Theorem 1] in the case of Kleinian groups and of Das-SimmonsUrbanski [DSU17, Theorem 1.2.1] in the case of Gromov hyperbolic spaces that

$$
\delta(\Gamma)=\operatorname{dim}_{\mathrm{H}}\left(\Lambda_{r}(\Gamma)\right) .
$$

The above immediately implies the upper bound $\delta(\Gamma) \leq \operatorname{dim}_{H}(\partial X)$. If $\Gamma$ is convexcocompact, then every limit point is radial so that

$$
\operatorname{dim}_{\mathrm{H}} \Lambda_{r}(\Gamma)=\operatorname{dim}_{\mathrm{H}} \Lambda(\Gamma) .
$$

In particular, if $\Gamma$ is a uniform lattice in $X$ then $\Lambda(\Gamma)=\partial X$ and

$$
\delta(\Gamma)=\operatorname{dim}_{\mathrm{H}} \partial X .
$$

Partial Poincare series. Let $\Gamma$ be a discrete subgroup of $\operatorname{Isom}(X)$. It will often be useful to consider a partial Poincare series, where the summation is performed over a subset of $\Gamma$. We are particularly interested in the following situation. Assume that the group $\Gamma$ is acting on a set $Z$. Given a point $z \in Z$ and a subset $Y \subset Z$ denote

$$
E_{\Gamma}(z, Y)=\{\gamma \in \Gamma: \gamma z \in Y\}
$$

Definition 2.1. The partial Poincare series of $\Gamma$ at the exponent $s \geq 0$ with respect to the given action of $\Gamma$ on $Z$ and the basepoint $o \in X$ is

$$
\mathcal{P}_{\Gamma}(s ; z, Y)=\sum_{\gamma \in E_{\Gamma}(z, Y)} e^{-s d_{X}(o, \gamma o)}
$$

where $z \in Z$ is any point and $Y \subset Z$ is any subset.

Clearly $\mathcal{P}_{\Gamma}(s ; z, Y) \leq \mathcal{P}_{\Gamma}(s)$ always holds, and we will be concerned with establishing useful estimates in the other direction.

Two different actions of $\Gamma$ will play a role below. Namely, either $Z=\operatorname{Isom}(X)$ and $V$ is an open subset (consisting of hyperbolic elements), or $Z$ is a Borel space with a probability measure $\mu$ and $Y$ is a Borel subset with $\mu(Y)>0$.

\section{Hyperbolic elements in the Poincare series}

Let $X$ be a proper geodesic Gromov hyperbolic metric space and $\Gamma$ a fixed uniform lattice in $\operatorname{Isom}(X)$.

Hyperbolic elements. An isometry $h \in \operatorname{Isom}(X)$ is hyperbolic if $h$ has exactly two fixed points $h^{+}, h^{-}$on the boundary $\partial X$ and $h$ is of infinite order. The points $h^{+}$and $h^{-}$are called the attracting and repelling points of the hyperbolic element $h$, respectively. We have that

$$
h^{n} x \rightarrow h^{+} \text {for all } x \in \partial X \backslash\left\{h^{-}\right\} \text {as } n \rightarrow \infty
$$

as well as the analogous property with $h^{+}$replaced by $h^{-}$and $h^{n}$ by $h^{-n}$.

Let $\mathcal{H}(X)$ denote the collection of all hyperbolic elements in $\operatorname{Isom}(X)$. The collection $\mathcal{H}(X)$ is clearly invariant under conjugation. There is a natural map

$$
\mathcal{E}: \mathcal{H}(X) \rightarrow \partial^{2} X, \quad \mathcal{E}(h)=\left(h^{-}, h^{+}\right) .
$$

It is easy to see that the map $\mathcal{E}$ is continuous. Moreover $\mathcal{E}$ is $\operatorname{Isom}(X)$-equivariant with respect to the conjugation action on $\mathcal{H}(X)$ and the diagonal action on $\partial^{2} X$ induced by the embedding $\operatorname{Isom}(X) \rightarrow \operatorname{Homeo}(\partial X)$.

Proposition 3.1. The collection $\mathcal{H}(X)$ of hyperbolic elements is open in $\operatorname{Isom}(X)$. 
Proof. Let $h \in \operatorname{Isom}(X)$ be a hyperbolic isometry with attracting and repelling points $\mathcal{E}(h)=\left(h_{-}, h_{+}\right) \in \partial^{2} X$. The compactness of $\partial X$ combined with the contraction property of the hyperbolic isometry $h$ allows us to choose two disjoint open neighborhoods $h^{-} \in U^{-}$and $h^{+} \in U^{+}$in $\partial X$ so that $U^{-} \cup U^{+} \neq \partial X$ and

$$
h^{N}\left(\partial X \backslash U^{-}\right) \subset U^{+} \text {and } h^{-N}\left(\partial X \backslash U^{+}\right) \subset U^{-}
$$

for some sufficiently large $N \in \mathbb{N}$.

The above condition defines an open subset of $\operatorname{Homeo}(\partial X)$ in the compact-open topology. Since the natural map $G \rightarrow \operatorname{Homeo}(\partial X)$ is continuous, this continues to hold for every element $g \in \operatorname{Isom}(X)$ in a sufficiently small neighborhood of $h$. We claim that any such $g$ must be hyperbolic as well.

For any point $\xi \in \partial X \backslash\left(U^{-} \cup U^{+}\right)$the condition $g^{m N} \xi \in U^{+}$holds for all $m \in \mathbb{N}$. In particular the isometry $g^{N}$, and therefore $g$ itself, is of infinite order.

It remains to rule out the possibility that the isometry $g$ is parabolic. In that case $g^{N}$ is clearly parabolic as well. Note that

$$
g^{m N}\left(\partial X \backslash U^{-}\right) \subset U^{+} \text {and } g^{-m N}\left(\partial X \backslash U^{+}\right) \subset U^{-}
$$

holds for all $m \in \mathbb{N}$. Suppose $g^{N}$ is parabolic. Let $p \in \partial X$ be the unique fixed point of $g^{N}$. For any point $\xi \in \partial X$ we would have both $g^{m N} \xi \rightarrow p$ and $g^{-m N} \xi \rightarrow p$ as $m \rightarrow \infty$. This contradicts the fact that $U^{-}$and $U^{+}$are disjoint. We conclude that $g$ is hyperbolic.

Proposition 3.2. Let $K \subset \mathcal{H}(X) \subset \operatorname{Isom}(X)$ be a compact subset. Then for any point $o \in X$ there is a radius $D=D(K, o)>0$ such that any bi-infinite geodesic line $l$ in $X$ with $l(-\infty)=h^{-}$and $l(\infty)=h^{+}$where $\left(h^{-}, h^{+}\right)=\mathcal{E}(h)$ for some $h \in K$ satisfies $l \cap B_{X}(o, D) \neq \emptyset$.

Proof. The definition of the visual metric on $\partial X$ implies that for any compact subset $Q \subset \partial^{2} X$ there is a compact $C \subset X$ such that any bi-infinite geodesic line $l$ in $X$ with endpoints $(l(-\infty), l(\infty)) \in Q$ satisfies $l \cap C \neq \emptyset$. The result now follows from the continuity of the map $\mathcal{E}: \mathcal{H}(X) \rightarrow \partial^{2} X$.

Partial Poincare series over conjugates of a hyperbolic element. Recall that given any element $g \in \operatorname{Isom}(X)$ and subset $U \subset \operatorname{Isom}(X)$ we denote

$$
E_{\Gamma}(g, U)=\left\{\gamma \in \Gamma: \gamma g \gamma^{-1} \in U\right\}
$$

and consider the associated partial Poincare series

$$
\mathcal{P}_{\Gamma}(s ; g, U)=\sum_{\gamma \in E_{\Gamma}(g, U)} e^{-s d_{X}(\gamma o, o)} .
$$

Lemma 3.3. Let $h \in \mathcal{H}(X)$ be a hyperbolic isometry and $K \subset \mathcal{H}(X)$ be a compact subset. Then for every exponent $s$ there is a constant $\beta=\beta(K, o, s)>0$ so that

$$
e^{-s d_{X}(\gamma o, o)} \leq \beta e^{-\frac{s}{2} d_{X}(h o, o)}
$$

for all elements $\gamma \in E_{\Gamma}(h, K)$.

Proof. Consider some element $\gamma \in E_{\Gamma}(h, K)$ so that in particular $\gamma^{-1} g \gamma=h$ for some hyperbolic isometry $g \in K$. The triangle inequality implies that

$$
\begin{aligned}
d_{X}(h o, o) & =d_{X}\left(\gamma^{-1} g \gamma o, o\right) \leq \\
& \leq d_{X}\left(\gamma^{-1} o, o\right)+d_{X}\left(\gamma^{-1} g o, \gamma^{-1} o\right)+d_{X}\left(\gamma^{-1} g \gamma o, \gamma^{-1} g o\right)= \\
& =2 d_{X}(\gamma o, o)+d_{X}(g o, o)
\end{aligned}
$$


We obtain the following estimate

$$
e^{-s d_{X}(\gamma o, o)} \leq e^{\frac{s}{2} d_{X}(g o, o)} e^{-\frac{s}{2} d_{X}(h o, o)}
$$

and the lemma follows with the constant $\beta$ being given by

$$
\beta=\sup _{g \in K} e^{\frac{s}{2} d_{X}(g o, o)} .
$$

The following property of uniform lattices is used in Lemma 3.5 below.

Lemma 3.4. Let $\Gamma$ be a uniform lattice in $\operatorname{Isom}(X)$ and $\mathrm{C}$ a compact subset of $X$. Then there is a constant $n=n(\Gamma, C)>0$ so that

$$
\left|\left\{\gamma \in \Gamma: \gamma g_{1} C \cap g_{2} C \neq \emptyset\right\}\right| \leq n
$$

for every pair of elements $g_{1}, g_{2} \in \operatorname{Isom}(X)$.

Proof. Since $\Gamma$ is a uniform lattice there is a compact subset $K \subset \operatorname{Isom}(X)$ satisfying $\Gamma K=\operatorname{Isom}(X)$. Denote $C^{\prime}=K C$. In particular $C^{\prime}$ is compact. Given any pair of elements $g_{1}, g_{2} \in \operatorname{Isom}(X)$ there are $\gamma_{1}, \gamma_{2} \in \Gamma$ so that $g_{i} \in \gamma_{i} K$ for $i \in\{1,2\}$. It suffices therefore to bound the size of the set

$$
\left\{\gamma \in \Gamma: \gamma \gamma_{1} C^{\prime} \cap \gamma_{2} C^{\prime} \neq \emptyset\right\} .
$$

Such an upper bound exists as $\Gamma$ is acting properly on $X$.

Lemma 3.5. Let $h \in \mathcal{H}(X)$ be a hyperbolic isometry and $K \subset \mathcal{H}(X)$ a compact subset. If $E_{\Gamma}(h, K) \neq \emptyset$ then there exists an element $\gamma_{h} \in E_{\Gamma}(h, K)$ such that

$$
\mathcal{P}_{\Gamma}(s ; h, K) \leq \alpha e^{-s d_{X}\left(\gamma_{h} o, o\right)}
$$

for some constant $\alpha=\alpha(K, \Gamma, o, s)>0$ depending on the exponent $s$ but independent of $h$.

Proof. It follows from Proposition 3.2 that there is a sufficiently large radius $D=$ $D(K, o)$ so that given any hyperbolic isometry $g \in K$, every bi-infinite geodesic line $l: \mathbb{R} \rightarrow X$ with endpoints equal to $\mathcal{E}(g)=\left(g^{-}, g^{+}\right)$satisfies $l(\mathbb{R}) \cap \mathrm{B} \neq \emptyset$ where we denote $\mathrm{B}=B_{X}(D, o)$.

Let $\mathrm{A}_{h} \subset X$ be any fixed bi-infinite geodesic in $X$ with endpoints equal to $\mathcal{E}(h)$. Every element $\gamma \in E_{\Gamma}(h, K)$ satisfies $\gamma h \gamma^{-1} \in K$ by definition. Therefore for every such $\gamma$ there exists a point $x_{\gamma} \in \mathrm{A}_{h}$ with $\gamma x_{\gamma} \in \mathrm{B}$. The triangle inequality implies that

$$
\begin{array}{r}
\mathcal{P}_{\Gamma}(s ; h, K)=\sum_{\gamma \in E_{\Gamma}(h, K)} e^{-s d_{X}(o, \gamma o)} \leq \sum_{\gamma \in E_{\Gamma}(h, K)} e^{-s\left(d_{X}\left(\gamma o, \gamma x_{\gamma}\right)-d_{X}\left(\gamma x_{\gamma}, o\right)\right)} \leq \\
\leq e^{s D} \sum_{\gamma \in E_{\Gamma}(h, K)} e^{-s d_{X}\left(o, x_{\gamma}\right)}
\end{array}
$$

for every exponent $s>0$.

Let $p \in \mathrm{A}_{h}$ denote a fixed nearest point projection of the point $o$ to $\mathrm{A}_{h}$. The thin triangles condition implies the existence of some $\kappa>0$, depending only on the hyperbolicity constant of $X$, such that given any other point $x \in \mathrm{A}_{h}$ we have

$$
d_{X}(x, o) \geq d_{X}(x, p)+d_{X}(p, o)-\kappa .
$$


This inequality and our previous estimate for the partial Poincare series give

$$
\mathcal{P}_{\Gamma}(s ; h, K) \leq e^{s\left(-d_{X}(p, o)+D+\kappa\right)} \sum_{\gamma \in E_{\Gamma}(h, K)} e^{-s d_{X}\left(x_{\gamma}, p\right)}
$$

for every exponent $s>0$. The series that appears in the last equation is essentially a geometric progression. To see this, we denote for every $r \in \mathbb{N}$

$$
E_{r}(h, K)=\left\{\gamma \in E_{\Gamma}(h, K): \exists x_{\gamma} \in \mathrm{A}_{h} \text { with } d\left(p, x_{\gamma}\right)<r \text { and } \gamma x_{\gamma} \in \mathrm{B}\right\} .
$$

Let $r_{0} \in \mathbb{N}$ be the minimal number so that $\left|E_{r_{0}}(h, K)\right|>0$ and choose an arbitrary element $\gamma_{h} \in E_{r_{o}}(h, K)$. In particular

$$
E_{\Gamma}(h, K)=\bigcup_{r \geq r_{0}} E_{r}(h, K) .
$$

According to Lemma 3.4 there is a number $n>0$ depending on the subset $K$, the subgroup $\Gamma$ and the basepoint $o$ but independent of the element $h$ so that

$$
\left|E_{r}(h, K) \backslash E_{r-1}(h, K)\right| \leq n
$$

for every $r \in \mathbb{N}$. We obtain

$$
\sum_{\gamma \in E_{\Gamma}(h, K)} e^{-s d_{X}\left(x_{\gamma}, p\right)} \leq \sum_{r \geq r_{0}} \sum_{\gamma \in E_{r}(h, K) \backslash E_{r-1}(h, K)} e^{-s r}<\alpha^{\prime} e^{-s r_{0}}
$$

where $\alpha^{\prime}$ is the constant $\alpha^{\prime}(K, \Gamma, o, s)=n\left(1-e^{-s}\right)^{-1}$. By the triangle inequality and the choice of $\gamma_{h}$ we have

$$
\begin{aligned}
d_{X}\left(\gamma_{h} o, o\right) \leq d_{X}\left(\gamma_{h} o, \gamma_{h} x_{\gamma_{h}}\right) & +d_{X}\left(\gamma_{h} x_{\gamma_{h}}, o\right) \leq d_{X}\left(o, x_{\gamma_{h}}\right)+D \leq \\
\leq & d_{X}(o, p)+d_{X}\left(p, x_{\gamma_{h}}\right)+D \leq d_{X}(o, p)+r_{0}+D .
\end{aligned}
$$

Putting everything together gives us

$$
\mathcal{P}_{\Gamma}(s ; h, K) \leq \alpha^{\prime} e^{s\left(-d_{X}(p, o)-r_{0}+D+\kappa\right)} \leq \alpha e^{-s d_{X}\left(\gamma_{h} o, o\right)}
$$

where $\alpha=\alpha(K, \Gamma, o, s)$ is the constant $\alpha=\alpha^{\prime} e^{s(2 D+\kappa)}$.

\section{Quantitative ReCurRence For aCtions of hyperbolic groups}

Let $X$ be a proper geodesic Gromov hyperbolic metric space and $G$ be a closed subgroup of Isom $(X)$ acting cocompactly on $X$ and admitting a uniform lattice. Fix an arbitrary basepoint $o \in X$.

Asymptotic notations. In what follows it will be convenient to introduce an asymptotic notation, writing $a \asymp_{c} b$ for some constant $c \geq 1$ to mean

$$
c^{-1} a \leq b \leq c a .
$$

Clearly $a \asymp_{c_{1}} b$ and $b \asymp_{c_{2}} d$ together imply $a \asymp_{c_{1} c_{2}} d$.

Similarly, if $\mu$ and $\nu$ are a pair of Borel measures on the same Borel space we will write $\mu \asymp_{c} \nu$ for some constant $c>1$ to mean that $\mu$ and $\nu$ are absolutely continuous with respect to each other and their Radon-Nykodim derivative satisfies

$$
\frac{d \mu}{d \nu} \asymp_{c} 1 .
$$


Growth of orbits. Let $\Gamma$ be a uniform lattice in $G$. Given a pair of positive radii $0 \leq r_{1} \leq r_{2}$ we let $A_{\Gamma}\left[r_{1}, r_{2}\right]$ denote the annulus

$$
A_{\Gamma}\left[r_{1}, r_{2}\right]=\left\{\gamma \in \Gamma: r_{1} \leq d_{X}(\gamma o, o) \leq r_{2}\right\} .
$$

It was shown by Coornaert that the critical exponent $\delta(\Gamma)$ controls the growth of annuli in the lattice $\Gamma$.

Theorem 4.1 (Coo93, Theorem 7.2]). For all sufficiently large $k>0$ there is a constant $a=a(k)>0$ with

$$
\left|A_{\Gamma}[r, r+k]\right| \asymp_{a} e^{\delta(\Gamma) r}
$$

for all $r \in \mathbb{N}$.

In fact Coornaert's theorem holds for any convex-cocompact subgroup.

The maximal ergodic theorem and quantitative recurrence. Fix a uniform lattice $\Gamma$ in the group $\operatorname{Isom}(X)$ as well as a Borel space $(Z, \mu)$ admitting an ergodic probability measure preserving action of $\Gamma$.

The following maximal ergodic theorem for probability measure preserving actions of lattices in Gromov hyperbolic spaces was recently obtained by Bowen and Nevo [BN13, Theorem 1.1].

Theorem 4.2 (Maximal ergodic theorem for hyperbolic groups). For all sufficiently large $k>0$ there is a constant $b=b(k)>0$ such that for any vector $f \in L^{2}(X, \mu)$ we have that

$$
\left\|\sup _{r \in \mathbb{N}} e^{-\delta(\Gamma) r} \sum_{g \in A_{\Gamma}[r, r+k]} g_{*} f\right\|_{2} \leq b\|f\|_{2} .
$$

We obtain the following straightforward quantitative recurrence result as a corollary of Theorem 4.2. Recall that given a Borel subset $U \subset Z$ and a point $x \in Z$ we denote

$$
E_{\Gamma}(x, U)=\{g \in \Gamma: g x \in U\} .
$$

Moreover for every $r, k>0$ let

$$
E_{r, k}(x, U)=E_{\Gamma}(x, U) \cap A_{\Gamma}[r, r+k] .
$$

Corollary 4.3 (Quantitive recurrence). For every sufficiently large $k>0$ there exists a constant $0<\kappa<1$ so that every Borel subset $U \subset Z$ with $\mu(U)>1-\kappa$ satisfies

$$
\mu\left(\left\{x \in Z: \inf _{r \in \mathbb{N}} e^{-\delta(\Gamma) r}\left|E_{r, k}(x, U)\right|>\kappa\right\}\right)>\kappa .
$$


Proof. Let $k>0$ be sufficiently large so that there are constants $a=a(k)$ and $b=b(k)$ as in Theorems 4.1 and 4.2. The maximal ergodic theorem implies that

$$
\begin{aligned}
b \mu\left(U^{c}\right) & \geq\left\|\sup _{r \in \mathbb{N}} e^{-\delta(\Gamma) r} \sum_{g \in A_{\Gamma}[r, r+k]} g_{*}\left(\chi_{X}-\chi_{U}\right)\right\|_{2} \geq \\
& \geq\left\|\sup _{r \in \mathbb{N}}\left(\frac{1}{a} \chi_{X}-e^{-\delta(\Gamma) r} \sum_{g \in A_{\Gamma}[r, r+k]} g_{*} \chi_{U}\right)\right\|_{2}= \\
& =\left\|\frac{1}{a} \chi_{X}-\inf _{r \in \mathbb{N}}\left(e^{-\delta(\Gamma) r} \sum_{g \in A_{\Gamma}[r, r+k]} g_{*} \chi_{U}\right)\right\|_{2} \geq \\
& \geq \frac{1}{a}\left\|\chi_{X}\right\|_{2}-\left\|\inf _{r \in \mathbb{N}}\left(e^{-\delta(\Gamma) r} \sum_{g \in A_{\Gamma}[r, r+k]} g_{*} \chi_{U}\right)\right\|_{2}
\end{aligned}
$$

We obtain

$$
\left\|\inf _{r \in \mathbb{N}} e^{-\delta(\Gamma) r} \sum_{g \in A_{\Gamma}[r, r+k]} g_{*} \chi_{U}\right\|_{2} \geq \frac{1}{a}-b \mu\left(U^{c}\right)>0
$$

provided that $\mu(U)>1-\kappa$ with $0<\kappa<1$ sufficiently small so that $a b \kappa<1$.

Corollary 4.4. There is a sufficiently small $\kappa>0$ such that every Borel subset $U \subset Z$ with $\mu(U)>1-\kappa$ satisfies

$$
\mu\left(\left\{x \in Z: \mathcal{P}_{\Gamma}(\delta(\Gamma) ; x, U)=\infty\right\}\right)>\kappa
$$

Proof. Let $k>0$ be sufficiently large and $\kappa>0$ sufficiently small as in Corollary 4.3. In particular, given any Borel subset $U \subset Z$ with $\mu(U)>1-\kappa$ there exists some Borel subset $Y \subset Z$ with $\mu(Y)>\kappa$ such that every point $x \in Y$ satisfies

$$
\inf _{r \in \mathbb{N}} e^{-\delta(\Gamma) r}\left|E_{r, k}(x, U)\right|>\kappa
$$

It follows that for every point $x \in Y$

$$
\begin{aligned}
\mathcal{P}_{\Gamma}(\delta(\Gamma) ; x, U) & =\sum_{\gamma \in E_{\Gamma}(x, U)} e^{-\delta(\Gamma) d_{X}(\gamma o, o)} \geq \\
& \geq \sum_{n \in \mathbb{N}} \sum_{\gamma \in E_{n k, k}(x, U)} e^{-\delta(\Gamma) d_{X}(\gamma o, o)} \geq \\
& \geq \sum_{n \in \mathbb{N}} e^{-\delta(\Gamma)(n+1) k}\left|E_{n k, k}(x, U)\right| \geq e^{-\delta(\Gamma) k} \sum_{n \in \mathbb{N}} \kappa=\infty
\end{aligned}
$$

as required.

\section{Discrete InVARIANT RANDOM SUbGroups}

Invariant random subgroups. Assume $X$ is a proper geodesic Gromov hyperbolic metric space. Let $G$ be a closed subgroup of $\operatorname{Isom}(X)$. We will use $\operatorname{Sub}(G)$ to denote the space of all closed subgroups of $G$ endowed with the Chabauty topology 1. Recall that the space $\operatorname{Sub}(G)$ is compact. The group $G$ admits a continuous action on $\operatorname{Sub}(G)$ by conjugation.

\footnotetext{
${ }^{1}$ This topology is generated by sub-basic Chabauty sets of the form $\{H \leq G$ closed : $H \cap U \neq \emptyset\}$ for all open subsets $U \subset G$ as well as of the form $\{H \leq G$ closed : $H \cap \bar{K}=\emptyset\}$ for all compact subsets $K \subset G$.
} 
An invariant random subgroup of $G$ is a conjugation invariant Borel probability measure on $\operatorname{Sub}(G)$. For example, every lattice $\Gamma$ in $G$ gives rise to an invariant random subgroup by pushing forward the $G$-invariant probability measure on $G / \Gamma$ to $\operatorname{Sub}(G)$ via the map $g \Gamma \mapsto g \Gamma g^{-1}$. More generally, given a normal subgroup $N$ in some lattice $\Gamma$ in $G$, we obtain an invariant random subgroup of $G$ by pushing forward the Dirac mass at the point $N \in \operatorname{Sub}(\Gamma)$ in the same way.

We say that an invariant random subgroup is discrete, of convergence or divergence type, geometrically dense, etc, if this property is satisfied on a $\mu$-conull set of closed subgroups. An invariant random subgroup $\mu$ is ergodic if the $G$-action on (Sub, $\mu$ ) is ergodic.

In the current work we are mostly interested in discrete invariant random subgroups. This turns out to be essentially always the case for semisimple linear groups over local fields.

Theorem 5.1 ([ABB 17, GL17 $)$. Let $G$ be a simple linear group over a local field . If $\mu$ is a non-atomic invariant random subgroup of $G$ then $\mu$ is discrete and Zariski dense.

Any group $G$ in Theorem 5.1 which has rank one is a closed cocompact subgroup in the group of isometries of a certain proper geodesic Gromov hyperbolic space, namely the corresponding rank one symmetric space or Bruhat-Tits tree.

The critical exponent of an invariant random subgroup. Let $\operatorname{DSub}(G)$ be the subspace of the Chabauty space $\operatorname{Sub}(G)$ consisting of discrete subgroups. The critical exponent $\delta(\mu)$ of the discrete invariant random subgroup $\mu$ is defined to be the expected value

$$
\delta(\mu)=\int_{\operatorname{DSub}(G)} \delta(\Gamma) \mathrm{d} \mu(\Gamma)
$$

The critical exponent $\delta(\mu)$ is well defined and lies in the closed interval $\left[0, \operatorname{dim}_{\mathrm{H}}(\partial X)\right]$ by Proposition A.3 of the appendix. If $\mu$ is an ergodic invariant random subgroup then $\delta(\Gamma)=\delta(\mu)$ for $\mu$-almost every discrete subgroup $\Gamma$ of $G$.

Discrete invariant random subgroups in Gromov hyperbolic spaces. Let $\Gamma$ be a uniform lattice in $G$. Recall that $\mathcal{H}$ denotes the subset of $G$ consisting of all hyperbolic elements. We assume that $G$ is non-elementary so that in particular $\operatorname{dim}_{\mathrm{H}}(\partial X)>0$.

Proposition 5.2. Let $\mu$ be an infinite invariant random subgroup of $G$. Then for every $0<\kappa<1$ there is an open relatively compact subset $V \subset \mathcal{H}$ such that

$$
\mu(\{H \leq G \text { closed }: H \cap V \neq \emptyset\})>1-\kappa .
$$

Proof. We first claim that $\mu$-almost every closed subgroup contains hyperbolic elements. Since $\mu$-almost every closed subgroup $H$ is infinite we may write the invariant random subgroup $\mu$ as a convex combination

$$
\mu=\theta \mu_{\text {parabolic }}+(1-\theta) \mu_{\text {non-parabolic }}
$$

for some $0 \leq \theta \leq 1$. Here $\mu_{\text {parabolic-almost every closed subgroup } H \text { has }}|\Lambda(H)|=1$

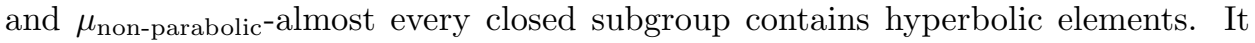
suffices to show that $\theta=0$.

\footnotetext{
${ }^{2}$ In the positive characteristic case Theorem 5.1 has an additional very mild technical assumption.
} 
Given a subgroup $H$ with $|\Lambda(H)|=1$ let $\lambda_{H} \in \partial X$ denote the unique limit point

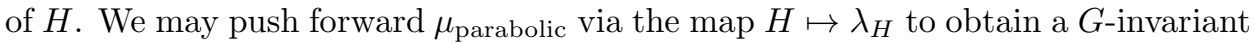
probability measure on the Gromov boundary of $X$. This is impossible, provided that $G$ itself is non-elementary. Therefore $\theta=0$ as required. Thus we have shown that $\mu$-almost every subgroup contains hyperbolic elements.

Finally note that the collection $\mathcal{H}$ of hyperbolic elements in $G$ is open by Proposition 3.1 and in particular can be exhausted by a countable collection of relatively compact open subsets. This completes the proof.

We are now ready to present the proof of one of our main results.

Theorem 5.3. Let $\mu$ be a discrete infinite invariant random subgroup of $G$. Assume that $G$ is non-elementary. Then

$$
\mathcal{P}_{\Delta}\left(\frac{\operatorname{dim}_{H}(\partial X)}{2}\right)=\infty
$$

for $\mu$-almost every closed subgroup $\Delta$ of $G$.

This implies of course that $\delta(\mu) \geq \frac{1}{2} \operatorname{dim}_{\mathrm{H}}(\partial X)>0$. In particular, since $\mu$ almost every subgroup contains hyperbolic elements $\mu$ must be non-elementary. The strict inequality $\delta(\mu)>\frac{1}{2} \operatorname{dim}_{\mathrm{H}}(\partial X)$ as in the statement of Theorem 1.1 will be established in $\$ 7$ below using Patteron-Sullivan theory.

Proof. The ergodic decomposition theorem allows us to write the invariant random subgroup $\mu$ as a convex combination of ergodic invariant random subgroups. We may therefore assume without loss of generality that $\mu$ is ergodic.

Fix a sufficiently small constant $0<\kappa<1$ so that Corollaries 4.3 and 4.4 hold true with respect to the probability measure preserving action of the lattice $\Gamma$ on the Borel space $(\operatorname{Sub}(G), \mu)$. Let $V \subset \mathcal{H} \subset G$ be an open relatively compact subset of hyperbolic elements as provided by Proposition 5.2. so that $\mu\left(\Omega_{V}\right)>1-\kappa$ where

$$
\Omega_{V}=\{H \leq G \text { closed : } H \cap V \neq \emptyset\} \subset \operatorname{Sub}(G) .
$$

To simplify notation let $\delta=\operatorname{dim}_{H}(\partial X)$. Observe that every element $h \in G$ with $E_{\Gamma}(h, V) \neq \emptyset$ is necessarily hyperbolic. According to Lemma 3.5 every such element $h$ satisfies

$$
e^{-\delta d_{X}\left(\gamma_{h} o, o\right)} \geq \frac{1}{\alpha} \mathcal{P}_{\Gamma}(\delta ; h, V)
$$

for some particular choice of $\gamma_{h} \in E_{\Gamma}(h, V)$, where $\alpha>0$ is a constant independent of $h$. We obtain the following estimate for the Poincare series $\mathcal{P}_{\Delta}(\delta / 2)$ of any discrete subgroup $\Delta$ of $G$

$$
\mathcal{P}_{\Delta}(\delta / 2)=\sum_{h \in \Delta} e^{-\frac{\delta}{2} d_{X}(h o, o)} \geq \frac{1}{\beta} \sum_{\substack{h \in \Delta \\ E_{\Gamma}(h, V) \neq \emptyset}} e^{-\delta d_{X}\left(\gamma_{h} o, o\right)} \geq \frac{1}{\alpha \beta} \sum_{h \in \Delta} \mathcal{P}_{\Gamma}(\delta ; h, V) .
$$

In the above estimate we used Lemma 3.3 to compare $d_{X}(h o, o)$ and $d_{X}\left(\gamma_{h} o, o\right)$, with the constant $\beta>0$ being as in that lemma.

The validity of the condition $\gamma \Delta \gamma^{-1} \in \Omega_{V}$ for some element $\gamma \in \Gamma$ implies by definition that $\gamma h \gamma^{-1} \in V$ for some $h \in \Delta$, and therefore

$$
\sum_{h \in \Delta} \mathcal{P}_{\Gamma}(\delta ; h, V) \geq \mathcal{P}_{\Gamma}\left(\delta ; \Delta, \Omega_{V}\right)
$$


According to Corollary 4.4 there is a Borel subset $Y \subset \operatorname{DSub}(G)$ with $\mu(Z)>\kappa$ and such that $\mathcal{P}_{\Gamma}\left(\delta ; \Delta, \Omega_{V}\right)=\infty$ for every subgroup $\Delta \in Y$. Therefore $\mathcal{P}_{\Delta}(\delta / 2)=\infty$ holds true for every $\Delta \in Y$ and hence $\mu$-almost surely by the ergodicity of $\mu$.

\section{QUASICONFORMAL DENSITIES}

Let $X$ be a proper geodesic Gromov hyperbolic metric space.

Busemann functions and shadows. We recall some basic definitions and facts.

Definition 6.1. Given any two points $x, y \in X$ the shadow from $x$ of the ball of radius $R>0$ around $y$ is

$$
\mathcal{S}_{R}(x, y)=\left\{\xi \in \partial X: \text { any geodesic ray from } x \text { to } \xi \text { intersects } B_{R}(y)\right\} .
$$

Fix a uniform lattice $\Gamma$ in $\operatorname{Isom}(X)$ and a basepoint $o \in X$.

Proposition 6.2. For all sufficiently large radii $k, R>0$ there is an integer $p \in \mathbb{N}$ so that for every $r>0$

$$
\bigcup_{\gamma \in A_{\Gamma}[r, r+k]} \mathcal{S}_{R}(o, \gamma o)=\partial X
$$

and moreover each ideal point $\xi \in \partial X$ belongs to at most $p$ of these shadows.

Proof. Let $D>0$ be a sufficiently large radius so that $\Gamma B_{X}(o, D)=X$. Consider an ideal point $\xi \in \partial X$ and let $l: \mathbb{R}_{\geq 0} \rightarrow X$ be any geodesic ray with $l(0)=o$ and $l(\infty)=\xi$. There exists some element $\gamma \in \Gamma$ so that $d_{X}(\gamma o, l(r+D)) \leq D$. Therefore $l\left(\mathbb{R}_{\geq 0}\right)$ intersects $B_{X}(\gamma o, 2 D)$. Recall that any other geodesic ray from $o$ to $\xi$ lies in the $\delta^{\prime}$ neighborhood of $l$ where $\delta^{\prime}$ is a constant. Taking $k=2 D$ and $R=2 D+\delta^{\prime}$, we find that any ideal point $\xi \in \partial X$ lies in some shadow $S_{R}(o, \gamma o)$ with $\gamma \in A_{\Gamma}[r, r+k]$ as required. Moreover, observe that any other shadow $S_{R}\left(o, \gamma^{\prime} o\right)$ with $\gamma^{\prime} \in A_{\Gamma}[r, r+k]$ and containing $\xi$ must satisfy $d_{X}\left(\gamma^{\prime} o, l(r+D)\right)<R+D$. As $\Gamma$ is a uniform lattice, the existence of an upper bound $p \in \mathbb{N}$ on the number of such shadows containing a given ideal point follows from Lemma 3.4 .

Proposition 6.3 (Coo93, Lemma 6.3]). Assume that $\operatorname{Isom}(X)$ admits a uniform lattice. Let $\rho_{o}$ be a visual metric on $\partial X$ with respect to the basepoint $o \in X$. Given a point $x \in X$ distinct from o let $\xi_{x} \in \partial X$ be the endpoint of any geodesic ray starting at $O$ and passing through $x$. Then

$$
\sup \left\{\rho_{o}\left(\zeta, \xi_{x}\right): x \text { distinct from o and } \zeta \in \partial X \backslash S_{R}(x, o)\right\}
$$

tends to 0 as $R \rightarrow \infty$.

Definition 6.4. The Busemann function $\beta_{\xi}: X \times X \rightarrow \mathbb{R}$ associated to the ideal point $\xi \in \partial X$ is given by

$$
\beta_{\xi}(x, y)=\lim \sup _{z \rightarrow \xi}\left(d_{X}(x, z)-d_{X}(y, z)\right)
$$

(here $z \in X$ is a point tending to $\xi$ along any geodesic ray with endpoint $\xi$ ).

Lemma 6.5 (Coo93, Lemma 6.2]). Let $x, y \in X$ be a pair of distinct points and $R>0$ a radius. Then for every $\xi \in \mathcal{S}_{R}(x, y)$ we have that

$$
d_{X}(x, y)-2 R \leq \beta_{\xi}(x, y) \leq d_{X}(x, y) .
$$


Quasiconformal densities. Consider a fixed non-elementary discrete subgroup $\Gamma$ of $\operatorname{Isom}(X)$. We use below the asymptotic notation introduced on p. 9 of 8 We use $\|\mu\|$ to denote the total mass a finite positive Borel measure.

Definition 6.6. $A \Gamma$-quasiconformal density of dimension $s \geq 0$ and distortion $d \geq 1$ is a family $\left\{\nu_{x}\right\}_{x \in X}$ of finite Borel measures on $\partial X$ such that

(1) the support of $\nu_{x}$ is the limit set $\Lambda(\Gamma)$ for all points $x \in X$,

(2) $\frac{d \nu_{x}}{d \nu_{y}}(\xi) \asymp_{d} e^{s \beta_{\xi}(y, x)}$ for $\nu_{y}$-almost every point $\xi \in \partial X$, and

(3) $g_{*} \nu_{x} \asymp_{d} \nu_{g x}$ for all elements $g \in \Gamma$ and points $x \in X$.

Recall that $\left(g_{*} \nu\right)(A)=\nu\left(g^{-1} A\right)$. As long as $\operatorname{dim}_{\mathrm{H}}(\partial X)<\infty$, any discrete subgroup $\Gamma$ admits a quasiconformal density of dimension $\delta(\Gamma)$. If $\Gamma$ is of divergence type then a $\Gamma$-quasiconformal density of dimension $\delta(\Gamma)$ is ergodic and essentially unique up to a bounded multiplicative factor, in the following sense.

Proposition 6.7. Assume that $\Gamma$ has divergence type and let $\left\{\nu_{x}\right\}_{x \in X}$ and $\left\{\eta_{x}\right\}_{x \in X}$ be two $\Gamma$-quasiconformal densities of the same dimension $s \geq 0$ and distortion $d \geq 1$. Then for all points $x \in X$

$$
\frac{\left\|\nu_{o}\right\|}{\left\|\eta_{o}\right\|} \asymp_{d^{4}} \frac{\left\|\nu_{x}\right\|}{\left\|\eta_{x}\right\|}
$$

Proof. According to MYJ15, Theorem 5.2] we have the estimate

$$
\frac{\nu_{o}(\xi)}{\eta_{o}(\xi)} \asymp_{d^{2}} \frac{\left\|\nu_{o}\right\|}{\left\|\eta_{o}\right\|}
$$

for all boundary points $\xi \in \partial X$. Combined with property (2) given in Definition 6.6 of quasiconformal densities this implies

$$
\frac{\nu_{o}(\xi)}{\nu_{x}(\xi)} \asymp_{d^{2}} \frac{\eta_{o}(\xi)}{\eta_{x}(\xi)} \Rightarrow \frac{\nu_{o}(\xi)}{\eta_{o}(\xi)} \asymp_{d^{2}} \frac{\nu_{x}(\xi)}{\eta_{x}(\xi)} \Rightarrow \frac{\left\|\nu_{o}\right\|}{\left\|\eta_{o}\right\|} \asymp_{d^{4}} \frac{\nu_{x}(\xi)}{\eta_{x}(\xi)} .
$$

The result follows by integrating the above estimate over all points $\xi$ in $\partial X$.

The following proposition allows us to relate quasiconformal densities of conjugate subgroups in a straightforward manner.

Proposition 6.8. Fix an element $g \in \operatorname{Isom}(X)$. If $\left\{\nu_{x}\right\}_{x \in X}$ is a $\Gamma$-quasiconformal density then $\left\{g_{*} \nu_{g^{-1}} x\right\}_{x \in X}$ is a $\left(g \Gamma g^{-1}\right)$-quasiconformal density of the same dimension and distortion.

Proof. Let $\left\{\nu_{x}\right\}_{x \in X}$ be a $\Gamma$-quasiconformal density of dimension $s \geq 0$ and distortion $d \geq 1$. Note that

$$
\frac{d g_{*} \nu_{g^{-1} x}}{d g_{*} \nu_{g^{-1} y}}(\xi)=\frac{d \nu_{g^{-1} x}}{d \nu_{g^{-1} y}}\left(g^{-1} \xi\right) \asymp_{d} e^{s \beta_{g^{-1}}\left(g^{-1} y, g^{-1} x\right)}=e^{s \beta_{\xi}(y, x)}
$$

Let $g \gamma g^{-1}$ be an arbitrary element of $g \Gamma g^{-1}$. Therefore

$$
\left(g \gamma g^{-1}\right)_{*} g_{*} \nu_{g^{-1} x}=(g \gamma)_{*} \nu_{g^{-1} x} \asymp_{d} g_{*} \nu_{\gamma g^{-1} x}=g_{*} \nu_{g^{-1}}\left(g \gamma g^{-1}\right) x
$$

It follows that $\left\{g_{*} \nu_{g^{-1}}\right\}_{x \in X}$ is a $\left(g \Gamma g^{-1}\right)$-quasiconformal density of the same dimension and distortion, as required. 
Remark 6.9. In the special case that the distortion is equal to one, a quasiconformal density is called a conformal density. In that case all of the above statements hold in a strict sense, and not just up to a multiplicative constant. This happens for example if $X$ is a CAT(-1)-space. However this assumption does not simplify the proof in any essential way.

\section{INVARIANT RANDOM SUBGROUPS OF DIVERGENCE TYPE}

Let $\mu$ be a discrete ergodic invariant random of divergence type.

The Poincare quasi-cocycle. There exists a $\mu$-measurable family $\nu$ of quasiconformal densities of dimension $\delta(\mu)$. This means that $\nu$ is a $\mu$-measurable map

$$
\nu: \operatorname{DSub}(G) \times X \rightarrow \mathcal{M}(\partial X), \quad \nu:(\Gamma, x) \mapsto \nu_{x}^{\Gamma}
$$

so that the family $\left\{\nu_{x}^{\Gamma}\right\}_{x \in X}$ is a $\Gamma$-quasiconformal density of dimension $\delta(\mu)$ and some fixed distortion $d \geq 1$ for $\mu$-almost every discrete subgroup $\Gamma$. We will additionally assume without loss of generality that the family $\nu$ is normalized so that

$$
\left\|\nu_{o}^{\Gamma}\right\|=\nu_{o}^{\Gamma}(\partial X)=1 .
$$

Since $\mu$ is of divergence type the existence of a $\mu$-measurable quasiconformal density is guaranteed by Proposition A.6 of the appendix.

Associated to the $\mu$-measurable quasiconformal density is a Poincare quasicocycle $\pi_{\nu}$. This is simply the map

$$
\pi_{\nu}: G \times \operatorname{DSub}(G) \rightarrow \mathbb{R}_{>0}, \quad \pi_{\nu}(g, \Gamma)=\left\|\nu_{g^{-1} o}^{\Gamma}\right\|=\nu_{g^{-1} o}^{\Gamma}(\partial X) .
$$

Since $\mu$ is of divergence type we have by Propositions 6.7 and 6.8 that

$$
g_{*} \nu_{g^{-1} x}^{\Gamma}{d^{2}}^{2} \frac{\left\|g_{*} \nu_{g^{-1} x}^{\Gamma}\right\|}{\left\|\nu_{x}^{g \Gamma g^{-1}}\right\|} \nu_{x}^{g \Gamma g^{-1}} \asymp_{d^{4}} \frac{\left\|g_{*} \nu_{g^{-1} o}^{\Gamma}\right\|}{\left\|\nu_{o}^{g \Gamma g^{-1}}\right\|} \nu_{x}^{g \Gamma g^{-1}}=\left\|\nu_{g^{-1}}^{\Gamma}\right\| \nu_{x}^{g \Gamma g^{-1}}
$$

for every element $g \in G$ and all points $x \in X$. In other words, the Poincare quasi-cocycle satisfies the relation

$$
g_{*} \nu_{g^{-1} x}^{\Gamma} \asymp_{d^{6}} \pi_{\nu}(g, \Gamma) \nu_{x}^{g \Gamma g^{-1}} .
$$

This implies that a cocycle relation holds for $\pi_{\nu}$ in an approximate sense, namely

$$
\pi_{\nu}(g h, \Gamma) \asymp_{d^{12}} \pi_{\nu}\left(g, h \Gamma h^{-1}\right) \pi_{\nu}(h, \Gamma)
$$

and in particular

$$
\pi_{\nu}\left(g^{-1}, g \Gamma g^{-1}\right) \pi_{\nu}(g, \Gamma) \asymp d^{12} 1
$$

for every pair of elements $g, h \in G$ and $\mu$-almost every closed subgroup $\Gamma$.

Remark 7.1. If every discrete subgroup of divergence type in $\operatorname{Isom}(X)$ admits a conformal (rather than quasiconformal) density then $\pi_{v}$ is a multiplicative cocycle in the usual sense. Whenever $G$ has Kazhdan's property $(T)$ any such cocycle is trivial Zim13, Theorem 9.1.1] and many of our arguments can be somewhat simplified. 
Sullivan's shadow lemma for invariant random subgroups. Consider a cocompact subgroup $G$ of $\operatorname{Isom}(X)$ and assume that $\mu$ is an ergodic discrete nonelementary invariant random subgroup of divergence type in $G$. Fix a $\mu$-measureable quasiconformal density $\nu$. In addition fix a basepoint $o \in X$.

Proposition 7.2 (Shadow lemma). Fix an arbitrary small constant $0<\kappa<1$. There is a Borel subset $Y \subset \operatorname{DSub}(G)$ with $\mu(Y)>1-\kappa$ such that for every sufficiently large radius $R>0$ there is a constant $c=c(R)>0$ with

$$
\nu_{o}^{\Gamma}\left(\mathcal{S}_{R}\left(o, g^{-1} o\right)\right) \asymp_{c} \pi_{\nu}(g, \Gamma) e^{-\delta(\mu) d_{X}(o, g o)}
$$

for every $g \in G$ and $\mu$-almost every closed subgroup $\Gamma$ with $g \Gamma g^{-1} \in Y$.

Proof. Recall that $\nu_{o}^{\Gamma}$ is normalized to be a probability measure and has full support on the limit set $\Lambda(\Gamma)$ for $\mu$-almost every subgroup $\Gamma$. Since $\mu$ is non-elementary the limit set $\Lambda(\Gamma)$ is $\mu$-almost surely not a single point and so

$$
\nu_{o}^{\Gamma}(\xi)<\nu_{o}(\partial X)=1
$$

for every ideal point $\xi \in \partial X$ and $\mu$-almost every subgroup $\Gamma$.

Let $\rho_{o}$ be a visual metric on the Gromov boundary $\partial X$ with respect to the basepoint $o \in X$. The compactness of $\partial X$ implies that there is a sufficiently small constant $\theta(\Gamma)>0$ depending on the subgroup $\Gamma$ such that

$$
\nu_{o}^{\Gamma}\left(\left\{\zeta \in \partial X: \rho_{o}(\zeta, \xi)>\theta(\Gamma)\right\}\right)>\theta(\Gamma)
$$

holds for every ideal point $\xi \in \partial X$. Since the family $\nu$ of quasiconformal densities is $\mu$-measurable the function $\theta$ can be chosen to be $\mu$-measurable on $\operatorname{DSub}(G)$. Moreover by Proposition 6.3 there is a constant $r(\Gamma)>0$ depending only on $\theta(\Gamma)$ so that for each point $x \in X$ there exists an ideal point $\xi \in \partial X$ with

$$
\left\{\zeta \in \partial X: \rho_{o}(\zeta, \xi)>\theta(\Gamma)\right\} \subset \mathcal{S}_{r}(x, o)
$$

for every radius $r>r(\Gamma)$. We may assume the function $r$ to be $\mu$-measurable.

Fix a pair of constants $\Theta>0$ sufficiently small and $R>0$ sufficiently large so that the following Borel set

$$
Y=\{\Gamma \in \operatorname{DSub}(G): \theta(\Gamma)>\Theta, r(\Gamma)<R\}
$$

satisfies $\mu(Y)>1-\kappa$.

Fix an element $g \in G$. Consider a discrete subgoup $\Gamma$ such that its conjugate $g \Gamma g^{-1}$ belongs to $Y$. In particular

$$
\nu_{o}^{g \Gamma g^{-1}}\left(\mathcal{S}_{R}(g o, o)\right)>\Theta .
$$

By the definition of the Poincare quasi-cocycle $\pi_{\nu}$ we have that

$$
\nu_{o}^{\Gamma}\left(\mathcal{S}_{R}\left(o, g^{-1} o\right)\right)=\nu_{o}^{\Gamma}\left(g^{-1} \mathcal{S}_{R}(g o, o)\right) \asymp_{d^{6}} \pi_{\nu}(g, \Gamma) \nu_{g o}^{g \Gamma g^{-1}}\left(\mathcal{S}_{R}(g o, o)\right) .
$$

where $d \geq 1$ is the distortion of the family $\nu$. To complete the proof of the shadow lemma we are required to estimate

$$
\begin{aligned}
\nu_{g o}^{g \Gamma g^{-1}}\left(\mathcal{S}_{R}(g o, o)\right) & =\int_{\mathcal{S}_{R}(g o, o)} \mathrm{d} \nu_{g o}^{g \Gamma g^{-1}}(\xi)= \\
& =\int_{\mathcal{S}_{R}(g o, o)} \frac{\nu_{g o}^{g \Gamma g^{-1}}(\xi)}{\nu_{o}^{g \Gamma g^{-1}}(\xi)} \mathrm{d} \nu_{o}^{g \Gamma g^{-1}}(\xi) \asymp_{d} \\
& \asymp_{d} \int_{\mathcal{S}_{R}(g o, o)} e^{-\delta(\nu) \beta_{\xi}(g o, o)} \mathrm{d} \nu_{o}^{g \Gamma g^{-1}}(\xi)
\end{aligned}
$$


where we have used the properties of quasiconformal densities in the final equation, see Definition 6.6. Finally, for every $\xi \in \mathcal{S}_{R}(g o, o)$ we have that

$$
d_{X}(g o, o)-2 R \leq \beta_{\xi}(g o, o) \leq d_{X}(g o, o)
$$

by Lemma 6.5. This concludes the proof with the constant

$$
c=d^{7} \Theta^{-1} e^{2 \delta(\mu) R} \text {. }
$$

Recurrence and the Poincare quasi-cocycle. Let $\Gamma$ be a uniform lattice in $G$. For every discrete subgroup $\Delta \in \operatorname{DSub}(G)$, Borel subset $Y \subset \operatorname{DSub}(G)$ and radius $r>0$ we let

$$
E_{r, k}(\Delta, Y)=E_{\Gamma}(\Delta, Y) \cap A_{\Gamma}[r, r+k]
$$

where $A_{\Gamma}[r, r+k]$ is an annulus in the group $\Gamma$ with respect to its action on $X$.

Proposition 7.3. Let $Y \subset \operatorname{DSub}(G)$ be the Borel subset with respect to which the shadow lemma (Proposition 7.2) holds. Then for all sufficiently large $k>0$ there is a constant $c^{\prime}>0$ such that

$$
\sum_{\gamma \in E_{r, k}(\Delta, Y)} \pi_{\nu}(\gamma, \Delta) \leq c^{\prime} e^{\delta(\mu) r}
$$

for every $r>0$ and $\mu$-almost every $\Delta \in \operatorname{DSub}(G)$.

Proof. According to Proposition 6.2 for all sufficiently large radii $k, R>0$ there is an integer $p$ so that

$$
\bigcup_{\gamma \in A_{\Gamma}[r, r+k]} \mathcal{S}_{R}(o, \gamma o)=\partial X
$$

for every $r>0$ and moreover every ideal point $\xi \in \partial X$ belongs to at most $p$ distinct shadows. We may assume that $R$ is sufficiently large for us to be able to use the Sullivan shadow lemma for invariant random subgroups. Let $c>0$ be the corresponding constant, as in Proposition 7.2

Denote $\Gamma_{r, k}=A_{\Gamma}[r, r+k]$. By the shadow lemma for invariant random subgroups

$$
\begin{aligned}
\frac{1}{c} e^{-\delta(\mu)(r+k)} \sum_{\gamma \in E_{r, k}(\Delta, Y)} \pi_{\nu}(\gamma, \Delta) & \leq \frac{1}{c} \sum_{\gamma \in E_{r, k}(\Delta, Y)} \pi_{\nu}(\gamma, \Delta) e^{-\delta(\mu) d_{X}(o, \gamma o)} \leq \\
& \leq \sum_{\gamma \in E_{r, k}(\Delta, Y)} \nu_{o}^{\Delta}\left(\mathcal{S}_{R}\left(o, \gamma^{-1} o\right)\right)=(*)
\end{aligned}
$$

for every radius $r>0$ and $\mu$-almost every closed subgroup $\Delta$. The estimate for $(*)$ can only go up when summing over all of $\Gamma_{r, k}$ rather than just $E_{r, k}(\Delta, Y)$, so that

$$
(*) \leq \sum_{\gamma \in \Gamma_{r, k}} \nu_{o}^{\Delta}\left(\mathcal{S}_{R}\left(o, \gamma^{-1} o\right)\right) \leq p \nu_{o}^{\Delta}\left(\bigcup_{\gamma \in \Gamma_{r, k}} \mathcal{S}_{R}\left(o, \gamma^{-1} o\right)\right)=p \nu_{o}^{\Delta}(\partial X)=p .
$$

The result follows with the constant $c^{\prime}=c p e^{\delta(\mu) k}$.

Proposition 7.4. Let $Y \subset \operatorname{DSub}(G)$ be any Borel subset. Then

$$
\int_{Y} \sum_{\gamma \in E_{r, k}(\Delta, Y)} \pi_{\nu}(\gamma, \Delta) \mathrm{d} \mu(\Delta) \geq \frac{1}{2 d^{12}} \int_{Y}\left|E_{r, k}(\Delta, Y)\right| \mathrm{d} \mu(\Delta)
$$

for every $r, k>0$, where $d$ is the distortion of the $\mu$-measurable family $\nu$. 
Proof. Denote $\Gamma_{r, k}=A_{\Gamma}[r, r+k]$. Given an element $\gamma \in \Gamma_{r, k}$ and a subgroup $\Delta \in Y$ it is clear that $\gamma \in E_{r, k}(\Delta, Y)$ if and only if $\Delta \in Y^{\gamma^{-1}} \cap Y$. This observation makes it possible to rewrite the expression on the left hand side of the inequality in the statement of the proposition, separating it into one summation over all unordered pairs of elements $\left\{\gamma, \gamma^{-1}\right\}$ with $\gamma^{2} \neq e$ and another summation over all elements $\gamma$ of order two, and finally switching the integral and the sum using Fubini's theorem. This gives

$$
\begin{aligned}
\sum_{\left\{\gamma, \gamma^{-1}\right\} \subset \Gamma_{r, k}, \gamma^{2} \neq e}\left(\int_{Y^{\gamma^{-1} \cap Y}} \pi_{\nu}(\gamma, \Delta) \mathrm{d} \mu(\Delta)+\int_{Y^{\gamma} \cap Y} \pi_{\nu}\left(\gamma^{-1}, \Delta\right) \mathrm{d} \mu(\Delta)\right) & = \\
& +\sum_{\gamma \in \Gamma_{r, k}, \gamma^{2}=e} \int_{Y^{\gamma} \cap Y} \pi_{\nu}(\gamma, \Delta) \mathrm{d} \mu(\Delta)=(*)
\end{aligned}
$$

Expression $(*)$ can be rearranged again by summing over all unordered subsets $\left\{\gamma, \gamma^{-1}\right\} \subset \Gamma_{r}$. Since every element of order two is accounted for twice we obtain

$$
(*) \geq \frac{1}{2} \sum_{\left\{\gamma, \gamma^{-1}\right\} \subset \Gamma_{r, k}} \int_{Y^{\gamma^{-1}} \cap Y}\left(\pi_{\nu}(\gamma, \Delta)+\pi_{\nu}\left(\gamma^{-1}, \Delta^{\gamma}\right)\right) \mathrm{d} \mu(\Delta)=(* *)
$$

Recall that $\pi_{\nu}$ is a multiplicative quasi-cocycle. In particular

$$
\pi_{\nu}\left(\gamma^{-1}, \gamma \Delta \gamma^{-1}\right) \pi_{\nu}(\gamma, \Delta) \asymp_{d^{12}} 1
$$

which immediately implies that

$$
\pi_{\nu}(\gamma, \Delta)+\pi_{\nu}\left(\gamma^{-1}, \Delta^{\gamma}\right) \geq 2 d^{-12}
$$

for every $\gamma \in \Gamma$ and $\mu$-almost every subgroup $\Delta$. The constant $d$ is the distortion of the $\mu$-measurable family $\nu$ of quasi-conformal densities. Proceeding with the estimate $(* *)$ we now obtain

$$
\begin{aligned}
(* *) & \geq \frac{1}{2} \sum_{\left\{\gamma, \gamma^{-1}\right\} \subset \Gamma_{r, k}} \int_{Y^{\gamma^{-1}} \cap Y} \frac{2}{d^{12}} \mathrm{~d} \mu(\Delta)=\frac{1}{d^{12}} \sum_{\left\{\gamma, \gamma^{-1}\right\} \subset \Gamma_{r, k}} \mu\left(Y \cap Y^{\gamma}\right) \geq \\
& \geq \frac{1}{2 d^{12}} \sum_{\gamma \in \Gamma_{r, k}} \mu\left(Y \cap Y^{\gamma}\right)=\frac{1}{2 d^{12}} \int_{Y}\left|E_{r}(\Delta, Y)\right| \mathrm{d} \mu(\Delta)
\end{aligned}
$$

as required.

\section{On the critical exponent of a divergence type invariant random subgroup.}

Theorem 7.5. Let $\mu$ be an discrete invariant random subgroup of divergence type in $G$. Then $\delta(\mu)=\operatorname{dim}_{H}(\partial X)$.

Proof. Recall that the critical exponent of any discrete subgroup of $\operatorname{Isom}(X)$ is bounded above by $\operatorname{dim}_{H}(\partial X)$. In particular $\delta(\mu) \leq \operatorname{dim}_{H}(\partial X)$ is true in general. We need to establish the reverse inequality, namely show that $\delta(\mu) \geq \operatorname{dim}_{H}(\partial X)$.

The ergodic decomposition allows us to assume from now on and without loss of generality that $\mu$ is ergodic. Since $\mu$ is of divergence type it must be infinite. Therefore Proposition 5.2 and Theorem 5.3 imply that $\mu$ is non-elementary.

We begin be setting up the required objects. Fix a basepoint $o \in X$ and let $\Gamma$ be a uniform lattice in $G$. Let $\nu$ be a $\mu$-measurable family of quasiconformal densities of dimension $\delta(\mu)$ and distortion $d \geq 1$, normalized so that $\left\|\nu_{o}^{\Delta}\right\|=1$. Let $\pi_{\nu}$ be the 
associated Poincare quasi-cocycle. Fix a sufficiently large $k>0$ and a sufficiently small constant $0<\kappa<1$ so that Corollary 4.3 of quantitative recurrence holds. Finally, let $Y \subset \operatorname{DSub}(G)$ be a Borel subset as in Proposition 7.2 (i.e. the shadow lemma for invariant random subgroups) and satisfying $\mu(Y)>1-\kappa$.

The quantitative recurrence property established in Corollary 4.3 provides us with a Borel subset $U \subset Y$ with $\mu(U)>0$ so that

$$
\left|E_{r, k}(\Delta, U)\right| \geq \kappa e^{\delta(\Gamma) r}
$$

for all $r>0$ and for every $\Delta \in U$. Integrating this over $U$ gives

$$
\int_{U}\left|E_{r, k}(\Delta, U)\right| \mathrm{d} \mu(\Delta) \geq(\kappa \mu(U)) e^{\delta(\Gamma) r}
$$

for all $r>0$. On the other hand, by combining the two previous Propositions 7.3 and 7.4 we deduce that

$$
\int_{Y}\left|E_{r, k}(\Delta, Y)\right| \mathrm{d} \mu(\Delta) \leq\left(2 d^{12} c^{\prime} \mu(Y)\right) e^{\delta(\mu) r}
$$

for all $r>0$. Since $U \subset Y$ these two estimates can only be compatible for all sufficiently large $r$ provided that $\delta(\mu) \geq \delta(\Gamma)=\operatorname{dim}_{H}(\partial X)$.

Our proofs of Proposition 7.2 and Theorem 7.5 are inspired by the elegant expository paper by Quint Qui06, especially his Lemma 4.10 and Theorem 4.11.

\section{Proofs of the Main Results}

We now complete the proofs of all the results stated in the introduction.

Proof of Theorem 1.1. Let $G$ be a non-elementary group acting cocompactly on $X$ and $\mu$ a discrete and infinite invariant random subgroup of $G$. The second part of Theorem 1.1 follows immediately from Theorem 7.5. Moreover

$$
\mathcal{P}_{\Delta}\left(\frac{\operatorname{dim}_{H}(\partial X)}{2}\right)=\infty
$$

for $\mu$-almost every closed subgroup $\Delta$ of $G$ according to Theorem 5.3 . In particular $\delta(\Delta) \geq \frac{1}{2} \operatorname{dim}_{H}(\partial X)>0$. We claim that in fact $\delta(\Delta)>\frac{1}{2} \operatorname{dim}_{H}(\partial X)$ happens $\mu$ almost always. For if this is not the case then $\delta(\Delta)=\frac{1}{2} \operatorname{dim}_{\mathrm{H}}(\partial X)$ and in particular $\Delta$ is of divergence type $\mu$-almost surely. Therefore $\delta(\Delta)=\operatorname{dim}_{\mathrm{H}}(\partial X)$ holds $\mu$ almost surely by Theorem 7.5, which leads to a contradiction as $\operatorname{dim}_{H}(\partial X)>0$.

Proof of Corollary 1.2. Let $G$ be a rank-one simple Lie group and $X$ be the associated Gromov hyperbolic symmetric space. It is known Sul79, Rob03 that the action of any discrete subgroup $\Gamma$ on $\partial^{2} X$ is ergodic with respect to the Lebesgue measure class if and only if $\mathcal{P}_{\Gamma}\left(\operatorname{dim}_{H}(\partial X)\right)=\infty$. Moreover the geodesic flow on $\Gamma \backslash G$ is ergodic if and only if $\Gamma$ acts ergodically on $\partial^{2} X$. In particular, the ergodicity of the geodesic flow on $\Gamma \backslash G$ implies in general that $\Gamma$ has divergence type. The converse implication of the corollary follows from part (2) of Theorem 1.1.

Proof of Corollary 1.3. Let $G$ be a rank-one simple Lie group and $\mu$ be a nonatomic invariant random subgroup of $G$ so that $\mu$-almost every subgroup is torsionfree. We know that $\mu$-almost every subgroup is discrete and non-amenable, see e.g. Theorem 5.1 and the remarks following Theorem 5.3 . Our main result implies that $\delta(\mu)>\frac{1}{2} \operatorname{dim}_{\mathrm{H}}(\partial X)$. The corollary follows from the precise relationship between the two quantities $\delta(\Gamma)$ and $\lambda_{0}(\Gamma \backslash X)$ mentioned in the introduction. 
Proof of Corollary 1.4. Let $G$ be one of the groups mentioned in the statement and assume that $G$ has Kazhdan's property $(T)$. It is known by [Cor90, Theorem 4.4], Leu04, Theorem 2] and [CDS17, Corollary 1.4] that there is some $\delta_{c}>0$ such that every discrete subgroup $\Gamma$ of $G$ is either a lattice or satisfies $\delta(\Gamma)<\operatorname{dim}_{H}(\partial X)-\delta_{c}$.

Now let $\mu$ be a discrete invariant random subgroup of $G$ of divergence type. Theorem 1.1 implies that $\delta(\mu)=\operatorname{dim}_{H}(\partial X)$. We deduce that $\mu$-almost every closed subgroup of $G$ is a lattice. Since $\mu$ is ergodic it must essentially be the invariant random subgroup associated to some particular lattice $\Gamma$ of $G$, see [SZ94, Corollary 3.2] for Lie groups or [HT16, Corollary 5.6] in general.

Proof of Corollary 1.5. Let $(Z, \mu)$ be a Borel space admitting a probability measure preserving action of $G$. We obtain an invariant random subgroup $\bar{\mu}$ of $G$ by considering the $G$-equivariant stabilizer map

$$
\text { Stab }: Z \rightarrow \operatorname{Sub}(G), \quad z \mapsto G_{z}
$$

and taking $\bar{\mu}=\operatorname{Stab}_{*} \mu$. The corollary follows by applying Theorem 1.1 to $\bar{\mu}$, provided that the stabilizer of $\mu$-almost every point $z \in Z$ is assumed to be discrete and infinite.

Proof of Corollary 1.6. Let $\Gamma$ be a lattice in the isometry group $\operatorname{Isom}(X)$ of some proper Gromov hyperbolic space space $X$. Let $(Z, \mu)$ be a Borel $\Gamma$-space with an invariant probability measure. It is possible to induce the $\Gamma$-action from $Z$ to a probability measure preserving action of $G=\operatorname{Isom}(X)$ on some Borel space $(\bar{Z}, \bar{\mu})$. See [Zim13, p. 75] for details. The stabilizer $G_{z}$ of $\bar{\mu}$-almost every point $z \in \bar{Z}$ is conjugate to the stabilizer $\Gamma_{z^{\prime}}$ of some point in $z^{\prime} \in Z$ and is in particular discrete. The result follows from Corollary 1.5 .

\section{Appendix A. Measurability in the Chabauty Borel space}

This appendix is dedicated to various technical issues related to the Borel structure on $\operatorname{Sub}(G)$. In particular, we show that several notions and constructions used above, such as the critical exponent and quasiconformal densities, all depend measurably on the choice of a discrete subgroup.

In general, given a second countable locally compact group $G$ and an open subset $U \subset G$, the set $\{H \leq G: H \cap U \neq \emptyset\}$ is Chabauty open. The Borel structure on $\operatorname{Sub}(G)$ coming from the Chabauty topology is called the Effros Borel structure [Kec12, §12.C]. Given a countable basis $U_{n}$ for the topology of $G$, the Effros Borel structure is generated by the subsets $\left\{H \leq G: H \cap U_{n}=\emptyset\right\}$.

The first result of the appendix holds true for any second countable locally compact group $G$.

Proposition A.1. The subset $\operatorname{DSub}(G)$ of discrete subgroups in $\operatorname{Sub}(G)$ is Borel.

Proof. This follows immediately from the observation that

$$
\operatorname{DSub}(G)=\bigcap_{\left\{n \in \mathbb{N}: e \in U_{n}\right\}}\left\{H \leq G: H \cap\left(U_{n} \backslash\{e\}\right)=\emptyset\right\} .
$$

From now on we specialize the discussion to the situation where $X$ is a proper geodesic Gromov hyperbolic metric space with basepoint $o \in X$ and $G$ is a closed subgroup of $\operatorname{Isom}(X)$. Some of the notations that we use below depend implicitly on $o$. 
Proposition A.2. For every radius $R>0$ the function

$$
n_{R}: \operatorname{DSub}(G) \rightarrow \mathbb{N}, \quad n_{R}(\Gamma)=\left|\Gamma o \cap B_{X}(o, R)\right|
$$

is Borel.

Proof. For every $\varepsilon>0$ choose an arbitrary $\varepsilon$-separated and $2 \varepsilon$-covering subset $S_{\varepsilon}$ of $X$ containing the base-point $o$. For every radius $R>0$ denote

$$
n_{R, \varepsilon}(\Gamma)=\mid\left\{x \in S_{\varepsilon}: x \in B_{X}(o, R) \text { and } B_{X}(x, 2 \varepsilon) \cap \Gamma o \neq \emptyset\right\} \mid
$$

where $\Gamma \in \operatorname{DSub}(G)$ is a discrete subgroup. It follows from the definition of the Effros Borel structure that the function $n_{R, \varepsilon}$ is Borel for every $R, \varepsilon>0$. Note that

$$
n_{R}(\Gamma)=\limsup _{\varepsilon \rightarrow 0} n_{R, \varepsilon}(\Gamma)
$$

and therefore $n_{R}$ is Borel as well for every $R>0$.

Proposition A.3. The critical exponent function

$$
\delta: \operatorname{DSub}(G) \rightarrow\left[0, \operatorname{dim}_{\mathrm{H}}(\partial X)\right]
$$

is Borel and conjugation invariant.

Proof. By the previous proposition, the orbital counting function $n_{R}(\Gamma)=\mid \Gamma o \cap$ $B_{X}(o, R) \mid$ is Borel on $\operatorname{DSub}(G)$ for every $R>0$. It follows that the critical exponent

$$
\delta(\Gamma)=\liminf _{R \rightarrow \infty} \frac{1}{R} \ln n_{R}(\Gamma)
$$

is a Borel measurable function on $\operatorname{DSub}(G)$ as well. The fact that $\delta$ is conjugation invariant is well known. To see this, it suffices to observe that

$$
\left|\Gamma o \cap B_{X}(o, R)\right|=\left|\left(g \Gamma g^{-1}\right) g o \cap B_{X}(g o, R)\right|
$$

and recall that the critical exponent is independent of the choice of basepoint.

Let $\mathcal{M}(X \cup \partial X)$ denote the convex space of probability measures on $X \cup \partial X$ with the weak-* topology. Given $\nu \in \mathcal{M}(X \cup \partial X)$ denote $\|\nu\|=\nu(X \cup \partial X)$. Let $\mathcal{M}_{1}(X \cup \partial X)$ denote the compact convex subset of $\mathcal{M}(X \cup \partial X)$ consisting of probability measures, that is all $\nu$ with $\|\nu\|=1$. Given any real number $s \geq 0$ denote

$$
\operatorname{DSub}_{>s}(G)=\delta^{-1}((s, \infty))=\{\Gamma \in \operatorname{DSub}(\Gamma): \delta(\Gamma)>s\} .
$$

In particular $\operatorname{DSub}_{>s}(G)$ is a Borel subset by the previous proposition.

Proposition A.4. For every real number $s \geq 0$ the map

$$
W_{s}: \operatorname{DSub}_{>s}(G) \rightarrow \mathcal{M}_{1}(X \cup \partial X), \quad W_{s}: \Gamma \mapsto \frac{\sum_{g \in \Gamma} e^{-s d_{X}(g o, o)} \delta_{g o}}{\sum_{g \in \Gamma} e^{-s d_{X}(g o, o)}}
$$

is Borel.

Proof. We proceed similarly to Proposition A.2 Namely, for every $\varepsilon$ choose an arbitrary $\varepsilon$-separated and $2 \varepsilon$-covering subset $S_{\varepsilon}$ of $X$ containing the basepoint $o$. Denote $\left.S_{\varepsilon}(\Gamma)=\left\{x \in S_{\varepsilon}: B_{X}(x),\right) \cap \Gamma \neq \emptyset\right\}$ and

$$
W_{s, \varepsilon}(\Gamma)=\frac{\sum_{x \in S_{\varepsilon}(\Gamma)} e^{-s d_{X}(x, o)} \delta_{x}}{\sum_{x \in S_{\varepsilon}(\Gamma)} e^{-s d_{X}(x, o)}} \in \mathcal{M}_{1}(X \cup \partial X)
$$

for every discrete subgroup $\Gamma \in \operatorname{DSub}_{>s}(G)$. By the definition of the Effros Borel structure the function $W_{s, \varepsilon}$ is Borel for all $\varepsilon$. Note that for every discrete subgroup 
$\Gamma \in \operatorname{DSub}_{>s}(G)$ the weak-* limit of the probability measures $W_{s, \varepsilon}(\Gamma)$ exists and in fact

$$
W_{s}(\Gamma)=\lim _{\varepsilon \searrow 0} W_{s, \varepsilon}(\Gamma) .
$$

The function $W_{s}$ is therefore Borel, as required.

Recall that the standard construction of a quasiconformal density for a fixed discrete group $\Gamma$ of divergence type involves taking a weak-* accumulation point as $s \searrow \delta(\Gamma)$. The following general lemma is needed to be able to do so in a measurable way.

Lemma A.5. Let $X$ be a Borel space, $M$ a second countable compact metric space and $f_{n}: X \rightarrow M$ a sequence of Borel maps. Then there is a Borel map $p: X \rightarrow M$ such that $p(x)$ is an accumulation point in $M$ of the sequence $f_{n}(x)$ for every $x \in X$.

Proof. Let $K(M)$ denote the Hausdorff space of all closed subsets of $M$. Let $P(x) \in$ $K(M)$ be the non-empty closed subset consisting of all the accumulation points of the sequence $f_{n}(x)$ for every $x \in X$. We claim that the map $P: X \rightarrow K(M)$ is Borel. The existence of the required selection function $p(x)$ will then follow at once from the Kuratowski-Ryll-Nardzewski selection theorem [Kec12, Theorem 12.13].

There is a countable family of open subsets $N_{m} \subset M$ for $m \in \mathbb{N}$ such that the Borel structure on $K(M)$ is generated by the Borel subsets $\{C \subset M$ closed : $\left.C \cap N_{m}=\emptyset\right\}$. Note that $P(x) \cap N_{m}=\emptyset$ is equivalent to $f_{n}(x) \in M \backslash N_{m}$ for all $n$ sufficiently large, which is a Borel condition.

Assume that $G$ is acting cocompactly on $X$ and admits a uniform lattice. We conclude appendix $\mathrm{A}$ by showing that a family of quasiconformal densities can be chosen in measurable way for all the discrete subgroups of a given critical exponent and having divergence type. Given any real number $\delta \geq 0$ denote

$$
\operatorname{DSub}_{\delta \text {-diver }}(G)=\{\Gamma \in \operatorname{DSub}(\Gamma): \delta(\Gamma)=\delta \text { and } \Gamma \text { is of divergence type }\} .
$$

Proposition A.6. For every $\delta \geq 0$ there is a constant $d \geq 1$ and a Borel map

$$
\nu: \operatorname{DSub}_{\delta \text {-diver }}(G) \times X \rightarrow \mathcal{M}(\partial X), \quad \nu:(\Gamma, x) \mapsto \nu_{x}^{\Gamma}
$$

so that $\left\{\nu_{x}^{\Gamma}\right\}_{x \in X}$ is a $\Gamma$-quasiconformal density of dimension $\delta$ and distortion $d$. Moreover we may normalize so that $\left\|\nu_{o}^{\Gamma}\right\|=1$ for all $\Gamma \in \operatorname{DSub}_{\delta \text {-diver }}(G)$.

Proof. Consider the Borel maps $W_{s}$ constructed in Proposition A.4 for every $s>\delta$. We may now apply Lemma A.5 to obtain

$$
\nu_{o}: \operatorname{DSub}_{\delta \text {-diver }}(G) \rightarrow \mathcal{M}(X \cup \partial X)
$$

which is a Borel map assigning to each discrete subgroup $\Gamma \in \operatorname{DSub}_{\delta \text {-diver }}(G)$ some weak-* accumulation point of the probability measures $W_{s}(\Gamma)$ as $s \searrow \delta$. We may now extend $\nu$ to a map defined on $\operatorname{DSub}_{\delta \text {-diver }}(G) \times X$ in a Borel measurable manner by insisting that the relation

$$
\frac{d \nu_{x}^{\Gamma}}{d \nu_{o}^{\Gamma}}(\xi)=e^{\delta \beta_{\xi}(o, x)}
$$

should be satisfied for every point $x \in X$. The collection $\left\{\nu_{x}^{\Gamma}\right\}_{x \in X}$ is indeed a $\Gamma$-quasiconformal density of critical exponent $\delta$ and distortion $d \geq 1$. These parameters depend only on the hyperbolicity constant of the Gromov space $X$ for every discrete subgroup $\Gamma \in \operatorname{DSub}_{\delta \text {-diver }}(G)$. See e.g. MYJ15, Lemma 6.1]. 


\section{REFERENCES}

$\left[\mathrm{ABB}^{+} 17\right]$ Miklos Abert, Nicolas Bergeron, Ian Biringer, Tsachik Gelander, Nikolay Nikolov, Jean Raimbault, and Iddo Samet. On the growth of $L^{2}$-invariants for sequences of lattices in Lie groups. Annals of math, 185(3):711-790, 2017.

[AC18] Goulnara Arzhantseva and Christopher Cashen. Cogrowth for group actions with strongly contracting elements. arXiv preprint arXiv:1803.05782, 2018.

[AGV14] Miklós Abért, Yair Glasner, and Bálint Virág. Kesten's theorem for invariant random subgroups. Duke Mathematical Journal, 163(3):465-488, 2014.

[BJ97] Christopher Bishop and Peter Jones. Hausdorff dimension and Kleinian groups. Acta Mathematica, 179(1):1-39, 1997.

[BK02] Mario Bonk and Bruce Kleiner. Rigidity for quasi-Moebius actions. Journal of Differential Geometry, 61(1):81-106, 2002.

[BN13] Lewis Bowen and Amos Nevo. von-Neumann and Birkhoff ergodic theorems for negatively curved groups. arXiv preprint arXiv:1303.4109, 2013.

[Can14] Jan Cannizzo. Schreier Graphs and Ergodic Properties of Boundary Actions. PhD thesis, Université d'Ottawa/University of Ottawa, 2014.

[CDS17] Rémi Coulon, Françoise Dal'bo, and Andrea Sambusetti. Growth gap in hyperbolic groups and amenability. arXiv preprint arXiv:1709.07287, 2017.

[Coo93] Michel Coornaert. Mesures de Patterson-Sullivan sur le bord dún espace hyperbolique au sens de Gromov. Pacific Journal of Mathematics, 159(2):241-270, 1993.

[Cor90] Kevin Corlette. Hausdorff dimensions of limit sets I. Inventiones mathematicae, 102(1):521-541, 1990.

[DSU17] Tushar Das, David Simmons, and Mariusz Urbański. Geometry and dynamics in Gromov hyperbolic metric spaces, volume 218. American Mathematical Soc., 2017.

[Els73a] Jürgen Elstrodt. Die resolvente zum eigenwertproblem der automorphen formen in der hyperbolischen ebene. teil i. Mathematische Annalen, 203(4):295-330, 1973.

[Els73b] Jürgen Elstrodt. Die resolvente zum eigenwertproblem der automorphen formen in der hyperbolischen ebene. teil ii. Mathematische Zeitschrift, 132(2):99-134, 1973.

[Els74] Jürgen Elstrodt. Die resolvente zum eigenwertproblem der automorphen formen in der hyperbolischen ebene. teil iii. Mathematische Annalen, 208(2):99-132, 1974.

[GKN12] Rostislav Grigorchuk, Vadim Kaimanovich, and Tatiana Nagnibeda. Ergodic properties of boundary actions and the Nielsen-Schreier theory. Advances in Mathematics, 230(3):1340-1380, 2012.

[GL17] Tsachik Gelander and Arie Levit. Invariant random subgroups over non-Archimedean local fields. arXiv preprint arXiv:1707.03578, 2017.

[HT16] Yair Hartman and Omer Tamuz. Stabilizer rigidity in irreducible group actions. Israel Journal of Mathematics, 216(2):679-705, 2016.

[Jae15] Johannes Jaerisch. A lower bound for the exponent of convergence of normal subgroups of Kleinian groups. The Journal of Geometric Analysis, 25(1):298-305, 2015.

[Kec12] Alexander Kechris. Classical descriptive set theory, volume 156. Springer Science \& Business Media, 2012.

[Kes59] Harry Kesten. Symmetric random walks on groups. Transactions of the American Mathematical Society, 92(2):336-354, 1959.

[Leu04] Enrico Leuzinger. Critical exponents of discrete groups and $l^{2}$-spectrum. Proceedings of the American Mathematical Society, 132(3):919-927, 2004.

[Mat05] Katsuhiko Matsuzaki. Isoperimetric constants for conservative Fuchsian groups. Kodai Mathematical Journal, 28(2):292-300, 2005.

[MYJ15] Katsuhiko Matsuzaki, Yasuhiro Yabuki, and Johannes Jaerisch. Normalizer, divergence type and Patterson measure for discrete groups of the Gromov hyperbolic space. arXiv preprint arXiv:1511.02664, 2015.

[Ōsh02] Kenichi Ōshika. Discrete groups, volume 207. American Mathematical Soc., 2002.

[Pat76] Samuel Patterson. The limit set of a Fuchsian group. Acta mathematica, 136(1):241273, 1976.

[Pat77] Samuel Patterson. Spectral theory and Fuchsian groups. In Mathematical Proceedings of the Cambridge Philosophical Society, volume 81, pages 59-75. Cambridge University Press, 1977. 
[Pat83] Samuel Patterson. Further remarks on the exponent of convergence of Poincaré series. Tohoku Math Journal, 35(2):357-373., 1983.

[Qui06] Jean-François Quint. An overview of Patterson-Sullivan theory. In Workshop The barycenter method, FIM, Zurich, 2006.

[Rob03] Thomas Roblin. Ergodicité et équidistribution en courbure négative. Société mathématique de France, 2003.

[Sul79] Dennis Sullivan. The density at infinity of a discrete group of hyperbolic motions. Publications Mathématiques de l'Institut des Hautes Études Scientifiques, 50(1):171202, 1979.

[Sul87] Dennis Sullivan. Related aspects of positivity in Riemannian geometry. Journal of differential geometry, 25(3):327-351, 1987.

[SZ94] Garrett Stuck and Robert J Zimmer. Stabilizers for ergodic actions of higher rank semisimple groups. Annals of Mathematics, pages 723-747, 1994.

[Zim13] Robert J Zimmer. Ergodic theory and semisimple groups, volume 81. Springer Science \& Business Media, 2013. 